\title{
Tracking Repeat Victimisation After Domestic Abuse Cases Are Heard With and Without Independent Domestic Violence Advisors (IDVAs) in an English Magistrate's Court
}

\author{
John Ross ${ }^{1} \cdot$ Jaqueline Sebire $^{2} \cdot$ Heather Strang ${ }^{3}$
}

Accepted: 24 January 2022 / Published online: 1 February 2022

(c) The Author(s) 2022

\begin{abstract}
Research Question Do cases heard in a specialist domestic abuse (SDA) court on days when Independent Domestic Violence Advisors (IDVAs) are present to engage with victims, compared to cases heard on days when no IDVAs are present, result in more convictions, or less frequency or severity of repeat victimisation?

Data This analysis included all 559 trials in one SDA court from June 2016 to December 2018, including 514 unique victims. IDVAs were present on the starting day of $84 \%$ of the trials, leaving $16 \%$ (90) cases to start on days when no IDVAs were present.
\end{abstract}

Methods The treatment and comparison cases were compared for similarity of 23 characteristics, with only one difference of over $20 \%$. The analysis proceeded as appropriate for a Level 4 (Sherman et al., Preventing crime: What works, what doesn't, what's promising: A report to the United States Congress, National Institute of Justice, 1997) quasi-experimental comparison between the treatment and comparison cases.

Findings IPA trials in the IDVA treatment group were $12 \%$ less likely than those in the comparison group to result in a conviction $(\mathrm{RR}=0.88,95 \% \mathrm{CI} 0.74-1.05)$. Trials in the IDVA treatment group had a $96 \%$ higher risk of being followed by a repeat domestic abuse incident in the 18 months after trial than trials in the no-IDVA comparison group $(R R=1.96,95 \% C I 1.19-3.23)$. Treatment group victims experienced a mean harm score for repeat victimisation in the 18 months post-trial eight times higher than the comparison group (80 compared with ten).

Conclusions The provision of Independent Domestic Violence Advisors in a specialist domestic abuse court was clearly correlated with higher rates of repeat victimisation, as well as higher levels of harm in repeat offences and lower rates of conviction. This correlation could well be causal, but only a randomised controlled trial can rule out that possibility.

Extended author information available on the last page of the article 
Keywords Domestic abuse courts · IDVAs · Intimate partner convictions · Cambridge Crime Harm Index (CCHI)

\section{Introduction}

The impact of domestic abuse on society is wide ranging, across economic, health and criminal justice systems. It is also hugely expensive: in 2019, the UK cost was estimated as $£ 66$ billion (Home Office, 2019). Yet the rate of holding perpetrators to account has been decreasing. In London, convictions for domestic abuse fell $20 \%$ in the year 2017-2018 to 2018-2019 (Police and Crime Committee, 2020). 'Evidential difficulties' are perceived as a major obstacle, primarily domestic abuse victims not supporting police action (Robinson \& Cook, 2006). It was to address this reluctance that in 2005, dedicated Magistrates' Courts called Specialist Domestic Abuse (SDA) Courts were created and the Independent Domestic Violence Advisor (IDVA) service introduced (Home Office, 2005).

IDVAs are specially trained and they operate independently of the criminal justice system. The stated purpose of IDVA services is to secure the safety of domestic abuse victims. They aim to serve as the primary point of contact for victims, operating as their advocates with other agencies and supporting safety planning for those at highest risk (SafeLives, 2014).

The provision of IDVA services is a key feature of the 2018 Government Victim Strategy to 'simplify the criminal justice experience and streamline support' (Home Office, 2018). Yet IDVA services operate outside of the statutory agency framework in the third sector and rely on commissioning decisions for funding. IDVA services are expensive, but often the funding is short-term and based on annual arrangements (Simmonds, 2019).

This study tracks convictions and repeat victimisations after the IDVA service was available in a London SDA Court, in comparison to similar cases in which IDVAs were not available. While the study is neither a randomised controlled trial nor a truly random "natural experiment" (Zeisel, 1973: 266), it does provide strong correlations between key outcomes and opportunities for IDVA engagement (or its absence) in similar kinds of cases. The objective is to track these outcomes to assess their implications for the need to have better evidence of the safety of IDVAs, and whether they provide any value for money.

\section{Almost a Natural Experiment}

In the SDA Court under study, a unique circumstance existed. All domestic abuse cases for this geographic area proceeding to prosecution are heard here, with the important exceptions of guilty plea cases and those deemed too serious to be dealt with in the Magistrate's Court. Because the case flow at this SDA Court was not sufficient to occupy a full week of the court's time, a multi-agency protocol was constructed to schedule most trials on Monday and Tuesdays, with Wednesday, Thursday and Friday available as overflow as need arises. The Court Listings Office 
coordinates all trial allocations for the court. Their system identified court space for all trials on a Monday or Tuesday, only allocating space outside of these days if there was insufficient space in advance of the trial time limits expiring. In allocating the trial date, the Listing Office have no information about the case, only its expected duration. No consideration can be given by staff to any other factors; for example, cases cannot be listed for trial on specific days because of the offence type, victim characteristics, etc.

One of the parties to the protocol is the IDVA service, which is available at the court only on Mondays and Tuesdays. Thus, the only difference in the court operation between Monday/Tuesday cases and the Wednesday/Thursday/Friday cases is the availability of the IDVA service. Although the case flow volumes are different during the week, the court does sit all week and its operation is the same all week. The pool of magistrates from which the Bench is chosen is the same, with the same Clerk staff and prosecuting staff on all 5 days a week.

This IDVA service engages with every victim who attends the Court on Mondays and Tuesdays and offers them advice and advocacy on the day. There is no prior contact with victims and no legacy contact, although signposting is provided to other services that can support thereafter. There is no contact with victims who do not attend for cases.

This operating arrangement provides the opportunity to explore whether the IDVA service enhances criminal justice effectiveness and keeps victims safer in the longerterm. This arrangement began in 2014 and provides an opportunity to be examined as a naturally occurring difference. Because the difference is not necessarily random, we refrain from calling it a truly natural experiment. Yet, the similarities in the cases provide strong elimination of many alternative explanations for the results. It therefore offers substantial cases for comparing IDVA engagement while tracking data on convictions and repeat victimisations.

In the Maryland Scale of Scientific Methods adopted by the Home Office (based on Sherman, et al., 1997), this study can claim to be a Level 4 analysis, in which multiple cases are matched on many criteria and compared in two groups that differ on one and only variable - in this case, the engagement of an IDVA. A Level 4 quasi-experiment offers the strongest causal inference of any quasi-experiment, and is the basis for many policy decisions in criminal justice.

\section{Data and Methodology}

This study includes all 559 domestic abuse trials at the SDA court in the period June 2016 to December 2018 (30 months), involving 514 unique victims. It notes every repeat domestic abuse matter reported against any of the victims in these trials in the 18-month period after the trial date, for a total of 196 repeat reports. The division of the cases into treatment and comparison groups based solely upon the opportunity for victims to receive the IDVA intervention provides reasonable levels of confidence of internal validity (Ruane, 2005) and care has been given to examine the extent of equivalence between the treatment and comparison groups. 
The primary dataset for the study is derived from the Crown Prosecution Service (CPS) system that records all cases listed at criminal courts in London. This was filtered to provide only the trials listed for the specific SDA Court, in the specified 30-month period subject of this research. The Court's unique reference number (URN) was used to identify the corresponding crime reference number issued by the police crime recording system, and that system was searched to identify all crime reports and offence details associated with the Court's URN. Data from all searchable data fields in the crime recording system was extracted for each report and added to the CPS system data to construct one detailed data set. These cases were then categorised as either intimate partner abuse (IPA) or family abuse (FA). FA cases, which comprised one fifth of all these domestic abuse trials, proved to be too few to subject to detailed analysis. The reported results therefore will track all domestic abuse trials for descriptive purposes (including FA cases) but focus primarily on IPA trials for the findings, with commentary added relating to FA findings where these were available.

The date of trial is contained in the data acquired from the CPS system and has been used to identify the day of the week the trial was heard. The data set has been coded to allow it to be ordered and analysed by treatment group (Monday/Tuesday) or comparison group (Wednesday/Thursday/Friday). The date of trial was also used to identify the subsequent 18 -month period, with the victims' personal details used to research the police crime recording system for domestic abuse reports made for that period; this was extracted and added to the data set.

Extensive efforts were then made to clean the data and exclude ineligible cases. The unit of analysis in this study is primarily individual events, either a trial or a repeat victimisation report, though there are some calculations in the analysis where it was judged to be important to employ victims as the unit of analysis. Although the data set includes victims who appear more than once, each victim has been included in these calculations only once so as not to distort the outcome.

After the removal of all ineligible cases, the data set comprised 559 trials and 514 victims, and 196 repeat reports for 92 repeat victims. A total of 90 of the 196 repeat reports (46\%) were incidents that did not meet the National Crime Recording Standards threshold to be recorded as a crime and were therefore recorded as a no-crime incident. Some victims were associated with one repeat report, some more than one (one victim was associated with 18 repeat reports). The analysis reviews all 559 trial events and 196 repeat events. Values are presented as percentages of the whole group rather than the two groups separately, to allow comparison between the two which inevitably have unequal volumes: only $16 \%$ of all trials were listed for comparison group days $(n=90)$, and only $15 \%$ of all victims $(n=79)$. It should be noted that definite confirmation that victims received IDVA input was not available. We could only assume that the opportunity to do so existed on the day of the trial, as required by policy and protocol.

\section{Results}

Using a comparison group to provide a baseline against which the treatment group findings are assessed requires a careful assessment of just how equivalent the two 
groups are in their composition. We have explained how cases are allocated to IDVA and non-IDVA days by the Court Listing Staff without information being available to them on any aspect of the cases beyond their likely duration.

To further examine the possibility of systemic factors leading to selection bias, the IPA treatment and comparison groups are presented in Tables 1 and 2 by a range of demographic and case severity factors to allow assessment of whether the two groups are sufficiently equivalent to allow reasonable comparison. Insufficient demographic data were available for FA cases, though where available they indicated patterns similar to the IPA cases.

The data set out in Tables 1 and 2, together with the description of how the Listings Office functions in allocating trial dates, support a conclusion that the allocation of trials was not subject to any detectable selection bias. The biggest difference is in the Table 1 difference in ethnicity of the accused, which is $53 \%$ in the comparison group and $31 \%$ in the treatment group, but out of 23 comparisons, one difference can be large simply by chance. The overall pattern suggests the findings to be interpreted as if any difference found between the groups could plausibly result from the presence or absence of IDVA support on the day of trial.

\section{Characteristics of the Cases (both IPA and FA)}

Overwhelmingly, most trials were for offences of violence $(75 \%, n=417)$, a category that comprises a diverse range of seriousness, ranging from assault without injury to serious wounding and threats to kill. Criminal damage was the second most common crime type at 5\% $(n=26)$. Most of the trial offences involved either no injury, minor injury or threats only $(95 \%, n=531)$. This is to be expected, because serious injury offences in English law are most likely to be of an offence classification requiring Crown Court rather than Magistrate Court-level scrutiny. 'Injury' is

Table 1 Intimate partner abuse (IPA) demographic characteristics by treatment and control groups

\begin{tabular}{llll}
\hline Characteristics & & Treatment group & $\begin{array}{l}\text { Com- } \\
\text { parison } \\
\text { group }\end{array}$ \\
\hline Victim gender & $\mathrm{F}$ & $93 \%$ & $89 \%$ \\
& $\mathrm{M}$ & $7 \%$ & $11 \%$ \\
Accused gender & $\mathrm{F}$ & $6 \%$ & $9 \%$ \\
& $\mathrm{M}$ & $94 \%$ & $91 \%$ \\
Victim mean age (yrs.) & & 34 & 37 \\
Accused mean age (yrs.) & & 37 & 37 \\
Victim ethnicity & White & $37 \%$ & $38 \%$ \\
& Black & $25 \%$ & $18 \%$ \\
& Asian & $28 \%$ & $30 \%$ \\
Accused ethnicity & White & $28 \%$ & $18 \%$ \\
& Black & $31 \%$ & $53 \%$ \\
& Asian & $30 \%$ & $22 \%$ \\
\hline
\end{tabular}


Table 2 IPA Case severity characteristics by treatment and comparison group

\begin{tabular}{llll}
\hline Characteristic & & Treatment $\%$ & Comparison $\%$ \\
\hline Offence - assault & & $77 \%$ & $62 \%$ \\
Trial offence risk grading & Standard & $58 \%$ & $52 \%$ \\
& Medium & $35 \%$ & $33 \%$ \\
Victim injury & High & $5 \%$ & $10 \%$ \\
& No & $42 \%$ & $49 \%$ \\
& Threats & $5 \%$ & $10 \%$ \\
& Minor & $48 \%$ & $36 \%$ \\
Victim is a vulnerable/intimidated witness & Moderate & $4 \%$ & $5 \%$ \\
Trial involves a repeat victim from a previous case & Serious & $1 \%$ & 0 \\
\hline
\end{tabular}

most usually interpreted as physical injury, with very few offences referring to psychological injury.

More than half of all cases were assessed by police using the Governmentapproved DASH risk assessment tool as 'standard' risk (no indicators of serious harm or their imminency identified by police) $(58 \%, n=323)$. A further $34 \%$ $(n=188)$ were assessed as medium risk (such indicators identified but not imminent) and $6 \%(n=31)$ as high risk (indicators identified and likely to be realised imminently). The focus of non-court IDVA services is entirely on victims assessed as high risk (SafeLives, 2014), so $94 \%$ of these cases involve victims who would not have had access to an IDVA service prior to the court trial date. Thus, the effect on a victim of accessing an IDVA service at this Court, or not, is largely free from the potential of contamination by any previous IDVA input.

In terms of the relationship status between the victim and the accused, $73 \%$ $(n=410)$ of the trials were intimate partner abuse, while $21 \%(n=115)$ were familial abuse. Most victims (86\%) were female, for whom the most frequent age banding was 30-39 years, while for the 14\% male victims, it was 19-29 years. The racial composition of all these victims closely reflected that of the Court services jurisdiction (see Table 1). In the 12 months prior to trial, $17 \%$ of all these victims $(n=86)$ had been a repeat victim of domestic abuse (18\% of trials involved a repeat victim, $n=100 / 559)$. In the 18 months after their trial, $18 \%$ $(n=92)$ reported repeat domestic abuse: $55 \%$ of these repeats $(n=51)$ reported one incident and $11 \%$ more than three. Almost half of these incidents $(46 \%$, $n=90$ ) did not meet Home Office guidelines for classification as a crime and were therefore classified as 'no-crime'.

In the results displayed below, unless otherwise specified, only IPA data are shown, owing to the paucity of data for FA cases. 


\section{Criminal Justice Effectiveness}

Trial 'effectiveness' is a measure of whether a trial went ahead on the scheduled date. Trials are lengthy hearings and consume court time. Listing trials that do not go ahead is inefficient and it unnecessarily delays genuine trials, with the added trauma of unnecessary delay for victims.

Figure 1 shows IPA trials in the treatment group were less likely than those in the comparison group to go ahead on the scheduled date $(R R=0.88,95 \%$ CI $0.81-0.96)$. In this analysis and all those following, 95\% confidence intervals (CI) are provided to show the range of likely true differences, with statistical significance established at 0.05 whenever the $C I$ range does not cross 1 (from a risk ratio above one to a ratio below one).

Figure 2 shows IPA trials in the treatment group were less successful at achieving a conviction than trials in the comparison group. Treatment group trials had a $12 \%$ reduction in likelihood of a conviction than the comparison group $(R R=0.88,95 \%$ CI 0.74-1.05) (In this case the difference is not significant, because the risk ratio crosses from below to above 1). Figure 3 illustrates significant and positive findings for IDVA-engaged family abuse cases however, where family abuse IDVA treatment group trials saw a $25 \%$ higher likelihood of conviction than the comparison group $(R R=1.25,95 \%$ CI $0.8-1.98)$.

\section{Count of Repeat Victimisation — Events (Intimate Partner Abuse Only)}

Figure 4 shows the difference between the two groups in repeat victimisation and shows the following:

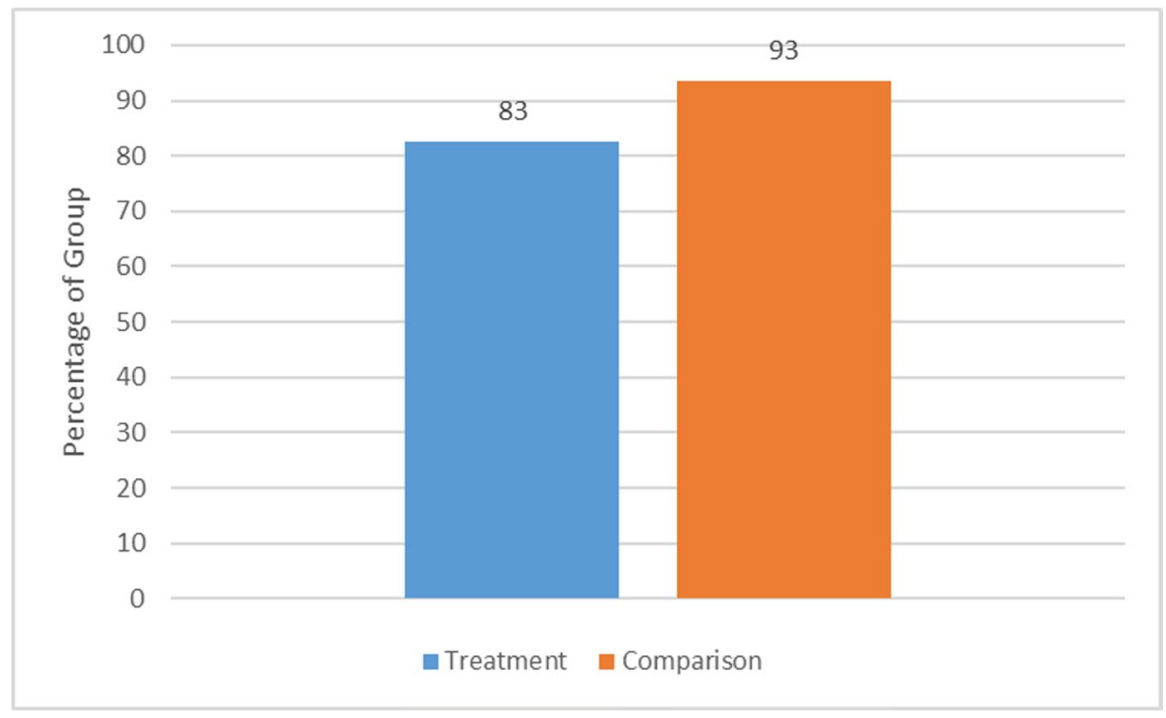

Fig. 1 Proportion of effective trials for intimate partner abuse 


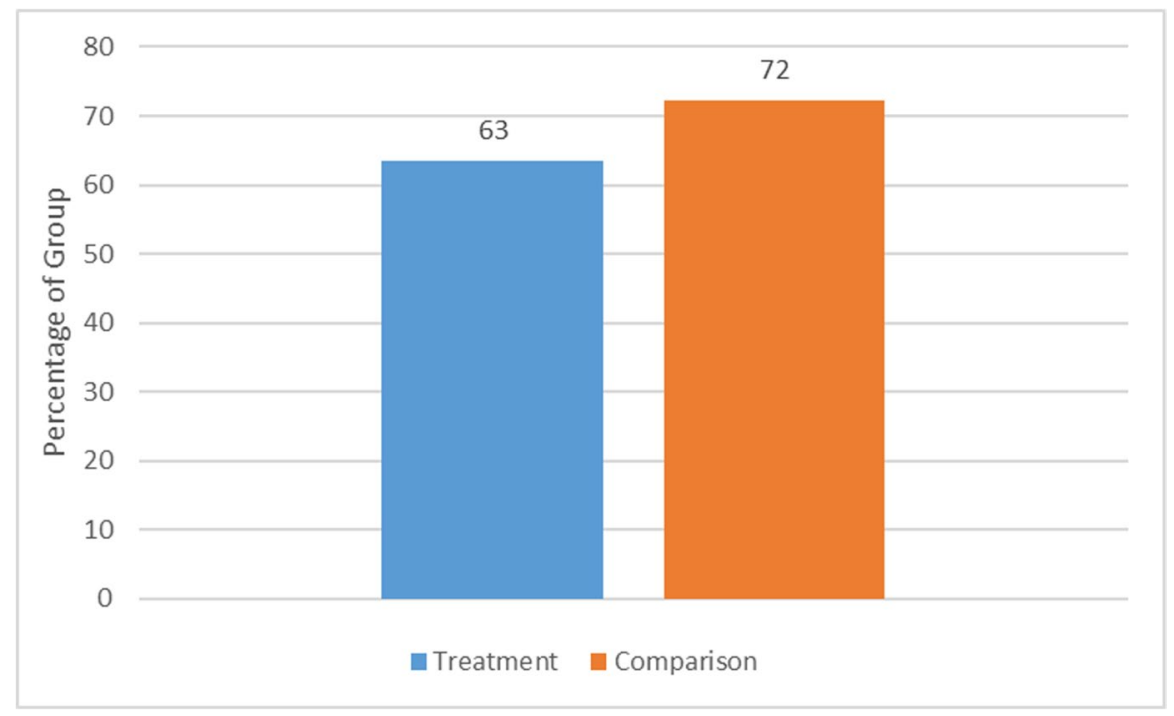

Fig. 2 Convictions on the day of trial for intimate partner abuse

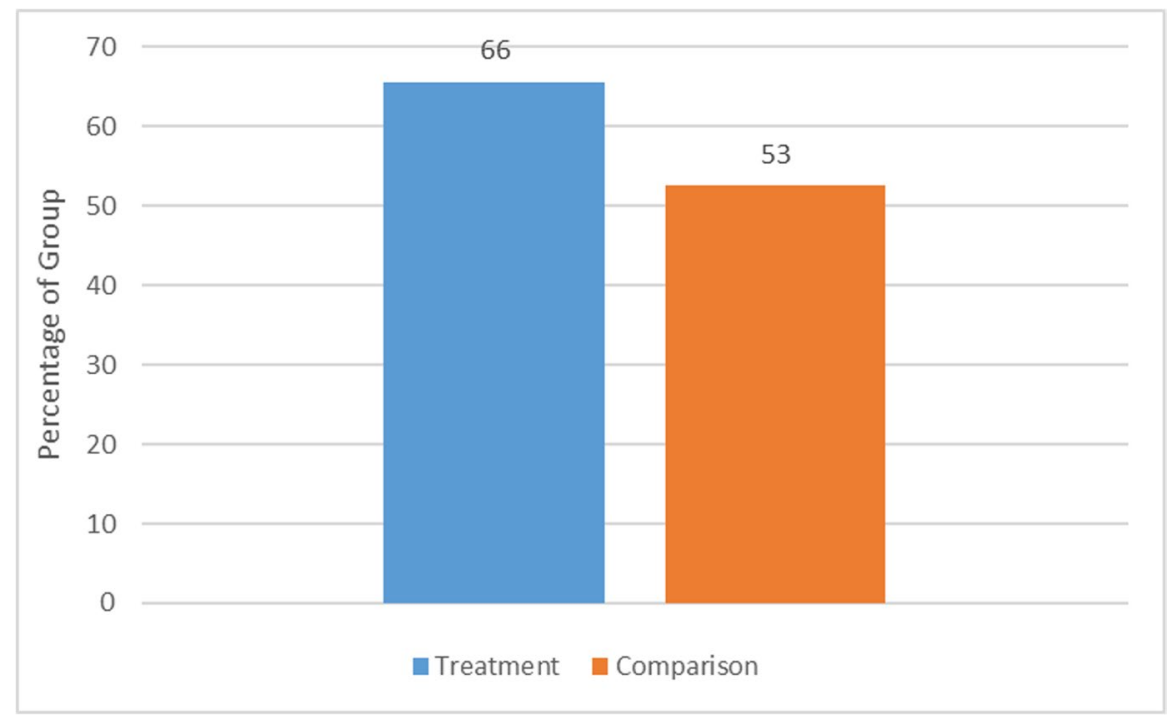

Fig. 3 Conviction on the day of trial for family abuse

- Trials in the IDVA treatment group had a $96 \%$ higher risk of being followed by a repeat domestic abuse incident in the 18-month after trial than trials in the noIDVA comparison group. This difference is both large and statistically significant $(R R=1.96,95 \%$ CI 1.19-3.23). 
- Compared to repeat incidents prior to the trials, more of the IDVA treatment group cases moved into repeat incident status after the trial than the non-IDVA comparison cases:

- Trials in the treatment group had a $110 \%$ increase in risk of resulting in a repeat domestic abuse incident after the trial than before. This is statistically significant $(R R=2.1,95 \%$ CI 1.68-2.75).

- Trials in the comparison group had a $30 \%$ increased risk of resulting in a repeat domestic abuse incident after the trial before. This is statistically significant $(R R=1.3,95 \%$ CI 0.62-2.73).

\section{Severity of Repeat Victimisation (Intimate Partner Abuse Only)}

The severity of the repeat victimisation assessed by application of the Cambridge Crime Harm Index (Sherman et al., 2016) is expressed as the mean number of days of imprisonment for each offence. All the trials concern crimes only and to ensure consistency in the calculation, only the crimes are included, but not the no-crime incidents, which do not attract a CCHI score.

Figure 5 shows the mean harm score in the treatment and comparison groups for IPA trials and repeat reports (these scores are identical for victims and events). Treatment group trial victims experienced eight times more harmful repeat victimisation in the 18-month after the trial than the comparison group.

Another way of looking at repeat victimisation severity is to calculate the percentage change in the treatment group repeat victimisation harm score post-trial against that of the treatment group cases at trial. By this interpretation, treatment group victims experienced a mean harm score post-trial $143 \%$ more harmful than

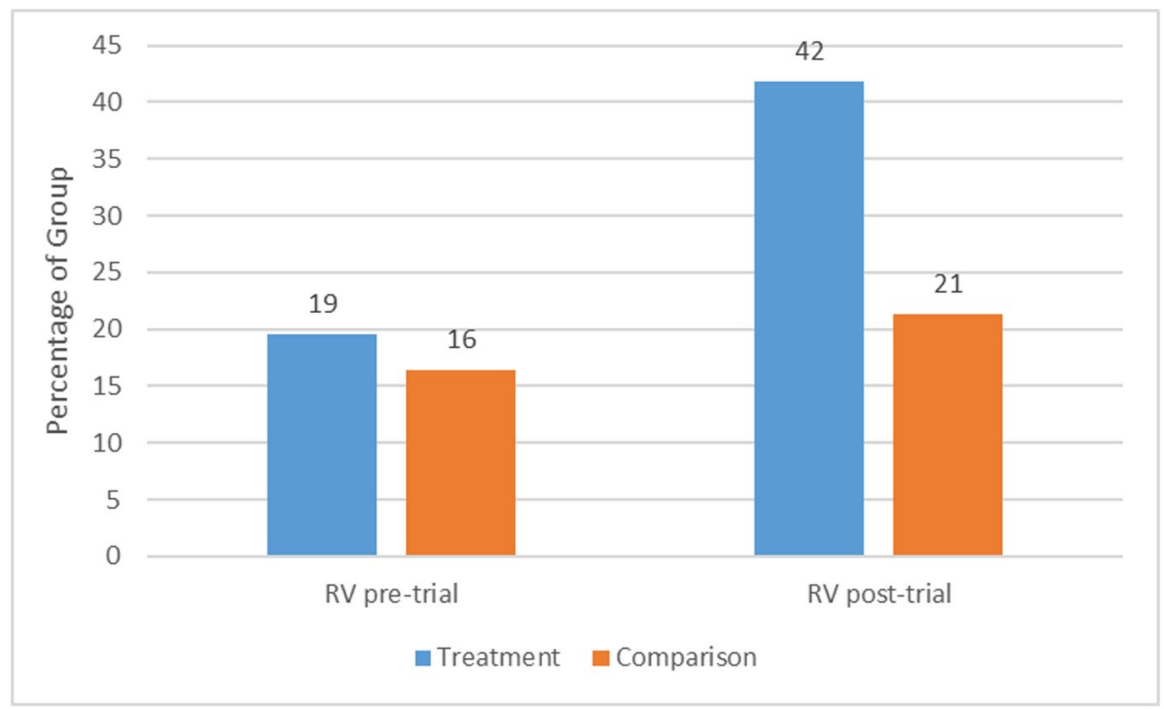

Fig. 4 Percentage of repeat victimisation for intimate partner abuse trials 
the case at trial (33 pre-trial compared with 80 post-trial). Furthermore, the corresponding figures for the comparison group were 99 and 10, a drop of $90 \%$.

Each of these analyses indicates that the IPA treatment group victims experience more reported harm post-treatment than the comparison group.

\section{Discussion}

The impact on attrition rates of domestic abuse victims not engaging with court prosecutions is well documented (Robinson \& Cook, 2006, Chopin \& Aebi, 2020). The IDVA role was created by the UK Government as a measure to address this issue (Home Office, 2005). Attrition in domestic abuse cases continues to be an important point of focus, as convictions in London declined $20 \%$ in the year ending 2019. Reducing the rate of attrition in cases of violence against women and girls is a Mayoral priority (Police and Crime Committee, 2020).

This study examines whether the support to victims of an IDVA service at court of the day of trials has a positive effect on the conviction rate and also on the rate and severity of repeat victimisation for the 18-month period thereafter. The research design employs a comparison group of similar cases which did not have the IDVA service and looks at three issues: trial completion effectiveness, frequency of repeat victimisation and severity of repeat victimisation.

The findings are stark. All of them defy the logic of the proposition that provision of IDVA opportunity makes things better. Few would predict that the opportunity for a victim to have the support of an IDVA at court would correlate to a lower risk of conviction, a higher risk of being a repeat victim or to victims experiencing more

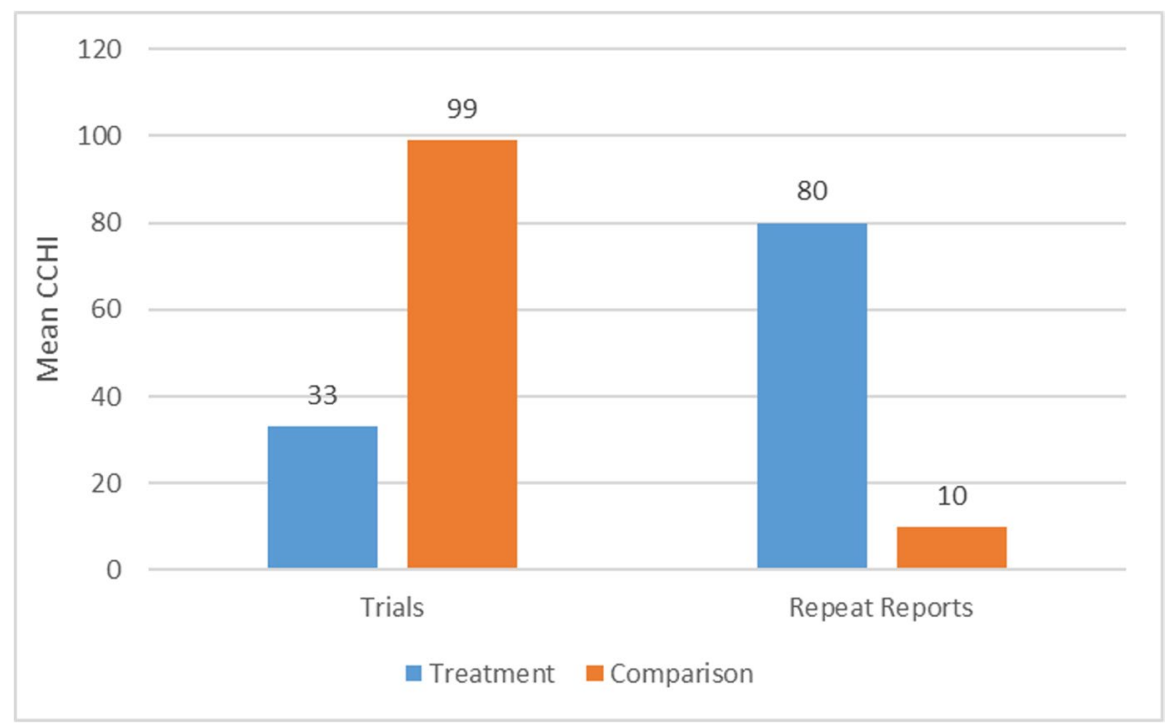

Fig. 5 Mean CCHI score for IPA trials and repeat reports 
harmful subsequent offending. Yet, the evidence in this research supports exactly those conclusions. This raises the question of how these results can be so different to those found in the initial evaluation of the IDVA pilots (Home Office, 2005).

A recent study found domestic abuse victims are most likely to retract their complaint in the 5-day period after a violent episode (Barrow-Grint, 2016), yet the victims in this research have persevered through that threshold, and the risk of conviction is still lower in the group with IDVA access on the day. Perhaps the potential identified by Morgan and Coombes (2013) for a conflict of purpose in the IDVA role, which measures victim satisfaction as its principal outcome measure, has led to the satisfaction value added not being associated with the three outcomes tested here.

By its research design, this study can only report on correlation not causation. While there is clearly a correlation between the opportunity for an IDVA and worse outcomes, that does not mean the opportunity for an IDVA causes those outcomes. That conclusion is beyond the reach of this research which is not a randomised controlled trial. The data have not been compiled within the rigour of a scientific framework and thus the conclusions to be drawn are far less certain. With this large caveat in mind, we summarise the answers to our three research questions as follows:

1. Does the opportunity for IDVA support at court on the day of trial have a positive effect on CJ effectiveness?

No. This study finds that trials are less effective and result in a lower rate of conviction in the IPA victim group with the IDVA benefit. This is concerning for policy makers as the IDVA provision is deployed as part of the specialist domestic abuse court (SDAC) for the explicit purpose of improving criminal justice effectiveness. It is possible that, as Morgan and Coombes (2013) suggest, there can be a conflict between the 'right' decision for the victim vs the 'right' decision for CJ effectiveness, but this study at least suggests that a major objective in setting up the IDVA service is not being met in respect of IPA victims.

2. Does the opportunity for IDVA support at court on the day of trial have a beneficial correlation with repeat incidents over the subsequent 18-month period?

No. Keeping victims safe is incontrovertibly part of the IDVA role responsibility as defined by SafeLives (2014) and this must include a reduction in the likelihood of repeat victimisation in the period after the IDVA service. But, this study finds at least a $10 \%$ increase in the risk of repeat victimisation for victims with the IDVA opportunity.

3. Does the opportunity for IDVA support at court on the day of trial have a beneficial correlation with reduced severity of abuse experienced by victims over the subsequent 18-month period, assessed by application of the Cambridge Crime Harm Index?

No. This research is unique by offering a means to evaluate whether a domestic abuse service has an effect on making a victim safer, as opposed to safe. It may be unrealistic in many domestic abuse relationships to hope that the abuse will stop because of any intervention, so perhaps a more useful measure is to aim for a 
reduction in the severity of harm. But, this study finds that IPA victims with IDVA benefit incur repeat victimisation that is notably more harmful than victims without IDVAs.

It is important to test hypotheses that challenge how the data are interpreted with these findings. Some of these are as follows:

1. The source data is recorded inaccurately leading to trials being attributed to an incorrect analysis group.

Compilation of the data was by manual input not system automation, so the potential for error exists. For virtually every data field examined, incomplete data was a feature, so the data set is indeed incomplete, but equivalently between the two groups. Only inaccuracy in the trial date (i.e. day of week) would be of concern here. But trials that did not have a date completed were not included in the data set.

2. The trial date allocation process is not accidental and leads to a systematically biased imbalance of case type between the analysis groups.

This is explored extensively in the assessment of equivalence above. The trial date allocation is made by the Listings Office staff who have no information about the cases other than the expected duration of the trial. The possibility for systemic bias has also been examined, and across all the data points available, no evidence of characteristic differences between the groups has been found in respect either of the victims or the offences.

3. An increase in reports of repeat victimisation is to be expected by victims who have had IDVA support as they are more confident to report.

The Home Office report (2005) stated the evaluation leading to the introduction of IDVAs found they delivered reduced repeat victimisation, not that repeat victimisation reports increased due to improved victim confidence. Reported domestic abuse offences in London increased 93\% between 2011 and 2019, due at least in part to improved police recording rather than improved victim confidence to report, in the view of the Police and Crime Committee (2020).

This claim is similar to that made by Davis et al. (2008) that there is no difference in repeat victimisation levels because of an advocate visit, but those who received the visit were inspired to report more. This is an interpretation rather than an evidential finding. Neither that study nor this one includes data to inform whether repeat incidents tend to be reported by the victim or a third party; this is important as the propensity of third parties to report incidents is beyond the influence of any confidence gained by an advocate's intervention. Neither this study nor Davis et al. can ascertain who made the reports and it is not possible to make a judgement on this claim without further information.

This study does, however, establish that regardless of the volume of repeat reports, they are on average far more harmful for the treatment group than the comparison group. 


\section{Implications for the Commissioners of IDVA Services}

This study raises the key question for commissioners: what objectives are sought from deployment of IDVAs? Specifically, as follows:

- Is the aspiration to keep victims safe or safer? What is the difference and how is it defined?

- By what data-supported metric should this be measured?

IDVA services have in the past been evaluated via qualitative feedback from recipients, with victim satisfaction as the main criterion i.e. measuring feelings of safety (Grandville \& Bridge 2010; Robinson, 2009; Howarth et al., 2009; Dheensa et al., 2020) rather than whether a victim was kept safe or safer, which is quite different. Simmonds (2019) suggests this is likely to be a consequence of the structure this sector takes in response to short-term/annual funding arrangements. Consideration could be given to provide the service within a statutory framework, to develop a long-term perspective on impact.

This research provides opportunity for commissioners to be challenged about what their objectives are in providing an IDVA service and importantly, through what evaluation framework should their achievement be assessed. But perhaps the whole domestic abuse landscape lacks a common definition of success, not just the IDVA sector, resulting currently in no common framework by which success can be judged.

\section{Implications for Policing}

There are two key considerations for policing identified from this study:

- The Home Office $(2004,2006)$ has estimated that $24 \%$ of all victims and witnesses within the CJ system are likely to be classed as vulnerable or intimidated and all domestic abuse victims are included in this category. Since 1999, these victims have all been entitled to 'special measures' (CPS, 2020), such as the right to give evidence from behind screens. There is evidence that victim/witnesses using these measures value them highly with as many as one third of those surveyed reporting they would otherwise have not given evidence (Burton et al., 2006; Hamlyn et al., 2004). Police are viewed as the agency with the knowledge and place in the CJ system to identify and submit the application for special measures. Most domestic abuse victims should be able to access this support, but this study finds that only $18 \%$ use it.

- In compiling this research, over a thousand police crime records were accessed and data extracted. Virtually, every record had fields with incomplete data. For research to become ever more important as police organisations engage more in understanding what works, the need for accurate and complete data becomes more important. Currently, poor data quality seriously limits evaluation capacity. 


\section{Conclusion}

Reporting to police of domestic abuse incidents continues to increase — up by $88 \%$ in 5 years 2012-2017 (Office for National Statistics, 2017). The mandate for effective working across the sector is clear. The perceived value of victim advocacy is long-established, having been a feature of criminal justice systems since the 1970s (Mawby \& Walklate, 1994).

This study examined 559 domestic abuse trials listed at a specialist domestic abuse court in London in 2016, 2017 and 2018. Cases heard on days with access to the IDVA service at court were found to have a $12 \%$ reduced likelihood of their accused abusers being convicted, a $10 \%$ increased risk of repeat victimisation and harm of repeat crimes $700 \%$ higher than victims whose cases were heard without that opportunity. The limitations of the study have been detailed, and the findings should be treated with caution. It does not conclude with any strong assertions about the value of IDVA services deployed in a court setting or more generally, but it does suggest a need for the sector to consider things might not be as thought or intended.

IDVA services are integral to the UK Government's strategic approach to victim care and reduced CJ attrition. There is a need for consideration of precisely what outcomes the service should deliver, how these should be evaluated and what structure is most appropriate to underpin delivery. Recognition should be given to the impact of short-term funding arrangements on the capacity for in-depth evaluation. Finally, the findings suggest the need for further research with RCT-level rigour to thoroughly evaluate the effect IDVA services have for victims, so that causation as well as correlation can be determined.

Open Access This article is licensed under a Creative Commons Attribution 4.0 International License, which permits use, sharing, adaptation, distribution and reproduction in any medium or format, as long as you give appropriate credit to the original author(s) and the source, provide a link to the Creative Commons licence, and indicate if changes were made. The images or other third party material in this article are included in the article's Creative Commons licence, unless indicated otherwise in a credit line to the material. If material is not included in the article's Creative Commons licence and your intended use is not permitted by statutory regulation or exceeds the permitted use, you will need to obtain permission directly from the copyright holder. To view a copy of this licence, visit http://creativecommons.org/licen ses/by/4.0/.

\section{References}

Barrow-Grint, K. (2016). Attrition rates in domestic abuse: Time for a change? An application of temporal sequencing theory. Policing, 10(3), 250-263.

Burton, M., Evans, R., \& Sanders, A. (2006). An evaluation of the use of special measures for vulnerable and intimidated witnesses. Home Office Findings, 270, 1-4.

Chopin, J., \& Aebi, M. F. (2020). (2018) 'The level of attrition in domestic violence: A valid indicator if the efficiency of a criminal justice system?' European Journal of Criminology, 17(3), 269-287.

CPS. (2020). Domestic abuse guidelines for prosecutors. Retrieved $15^{\text {th }}$ July 2020 from https://www.cps.gov.uk/ legal-guidance/domestic-abuse-guidelines-prosecutors.

Davis, R. C., Weisburd, D., \& Taylor, B. (2008). Effects of second responder programs on repeat incidents of family abuse. Campbell Systematic Reviews, 2008, 15. 
Dheensa, S., Halliwell, G., Daw, J., Jones, S. K., \& Feder, G. (2020). (2020) “From taboo to routine”: A qualitative evaluation of a hospital-based advocacy intervention for domestic violence and abuse.' BMC Health Services Research, 20, 129.

Hamlyn, B., Phelps, A., Turtle, J., \& Sattar, G. (2004) Are special measures working? Evidence from surveys of vulnerable and intimidated witnesses. Home Office Research Study 283, Development and Statistics Directorate.

Home Office. (2018). Victims strategy. HM Government, London HMSO.

Home Office. (2019). Ratification of the Council of Europe Convention on combating violence against women and girls and domestic violence (Istanbul convention) - 2019 report on progress. HMSO.

Howarth, E. et al. (2009). Safety in Numbers, a mulita-site evaluation of independent domestic violence advisor services. The Henry Smith Charity.

Mawby, R. I., \& Walklate, S. (1994). Critical victimology. Sage Publications.

Police and Crime Committee. (2020). 'London assembly: Domestic abuse.' April 2020.

Robinson, A. (2009). Independent domestic abuse advisors: A process evaluation. Cardiff University and funded by the Home Office.

Robinson, A., \& Cook, D. (2006). Understanding victim retraction in cases of domestic violence: Specialist courts, government policy, and victim-centred justice. Contemporary Justice Review, 9(2), 189-213.

Ruane, J. M. (2005). Essentials of research methods. Blackwell Publishing.

SafeLives. (2014). National definition of IDVA work. Retrieved 2 July 2020 from: https://safelives.org.uk/sites/ default/files/resources/National\%20definition\%20of\%20IDVA\%20work\%20FINAL.pdf.

Sherman, L. W., Gottfredson, D. C., MacKenzie, D. L., Eck, J., Reuter, P., \& Bushway, S. (1997). Preventing crime: What works, what doesn't, what's promising: A report to the United States Congress. National Institute of Justice.

Simmonds, L. (2019). The impact of local commissioning on victim services in England and Wales: An empirical study. International Review of Victimology, 25(2), 181-199.

Sherman, L. W., Neyround, P. W., \& Neyroud, E. (2016). The Cambridge Crime Harm Index: Measuring total harm for crime based on sentencing guidelines. Policing: A Journal of Policy and Practice, 10(3), 171-183.

Zeisel, H. (1973). Reflections on experimental techniques in the law. The Journal of Legal Studies, 2(1), $107-124$.

Publisher's Note Springer Nature remains neutral with regard to jurisdictional claims in published maps and institutional affiliations.

\section{Authors and Affiliations}

\section{John Ross ${ }^{1} \cdot$ Jaqueline Sebire $^{2} \cdot$ Heather Strang ${ }^{3}$}

$\triangle$ Heather Strang

hs404@cam.ac.uk

1 Metropolitan Police Service, London, UK

2 Bedfordshire Constabulary, Bedford, UK

3 University of Cambridge, Jerry Lee Centre of Experimental Criminology, Cambridge, UK 